\title{
Enhancing Marker-Based AR Technology
}

Jonghoon Seo, Jinwook Shim, Ji Hye Choi, James Park, and Tack-don Han

\author{
134, Sinchon-dong, Seodaemun-gu, Seoul, Korea \\ \{jonghoon. seo, jin99foryou, asellachoi, \\ james.park, hantack\} @msl.yonsei.ac.kr
}

\begin{abstract}
In this paper, we propose a method that solves both jittering and occlusion problems which is the biggest issue in marker based augmented reality technology. Because we adjust the pose estimation by using multiple keypoints that exist in the marker based on cells, we can predict the strong pose on jittering. Additionally, we can solve the occlusion problem by applying tracking technology.
\end{abstract}

Keywords: Marker-based AR, Augmented Reality, Tracking.

\section{Introduction}

Augmented reality(AR) technology combines digital information with real environment and provides insufficient information in real world.[1] Unlike virtual reality(VR) technology which substitutes the real environment with the graphics produced by a computer, AR technology annotates onto the real environment [2].

In order to implement augmented reality technology, many component technologies are needed - Detection, Registration, Tracking and so on. To implement practically, Registration and Tracking technology should be researched. Marker based tracking which uses visually fiducial marker in real world, offers more robust Registration and Tracking quality. Although this technology uses visually obtrusive marker in real world, it can offer more precise and faster tracking quality. So it is used in various commercial AR applications.

Conventional marker-based tracking, however, has used minimum number of features to estimate camera pose, so it is fragile by noise. Also, it has performed detection in every frame without tracking technology. So it could not perform augmenting when detection is failed. In this paper, to overcome these marker-inherent problems, we propose a hybrid method with marker-less tracking technology. In marker-less tracking, multiple features are used to estimate camera pose, so it provides more robust estimation than marker-based tracking. Also, marker-less tracking adopts tracking technology, it can continue augmenting even detectionfailure condition. In this paper, we adopt those marker-less tracking technology in marker-based tracking technology. We used multiple feature points in marker-based tracking to provide more robust against noise, and implemented feature-tracking method to avoid detection failure condition. 
In chapter 2, the related existing marker-based AR technology is described. We will explain the proposed method in chapter 3 and method which solves the jittering problem in chapter 3.1. We will explain the method that can solve occlusion problem in chapter 3.2. Chapter 4 and 5 shows the results obtained though experiments using our method and conclusion, in respectively.

\section{Related Works}

The study of augmented reality marker based study was researched actively because of the advantages of correctness and the speed. The marker that was developed with various type of shape such as circle or LED finally settled to the squared typed marker and it has been researched from many researchers after Matrix[3] \& ARToolkit[4] was developed. These markers calculate recognizes the contour of the square and identify it as the marker after the inside of the pattern is acceptable. It uses the augmenting 3D object method with the 3D pose estimation by calculating the perspective distortion of four vertexes. For these markers, the ARToolKit[4] that was invented at HITLab is most famous in HCI field. ARTag[5] was developed with improving the ARToolKit's recognition performance. In addition, T.U.Graz developed the Unobtrusive Marker[6] and solved the unrealistic problem with the marker. GIST developed the Simple Frame Marker[7] to increase the readability of marker. However, these researches have a purpose to improve the performance of the marker itself.

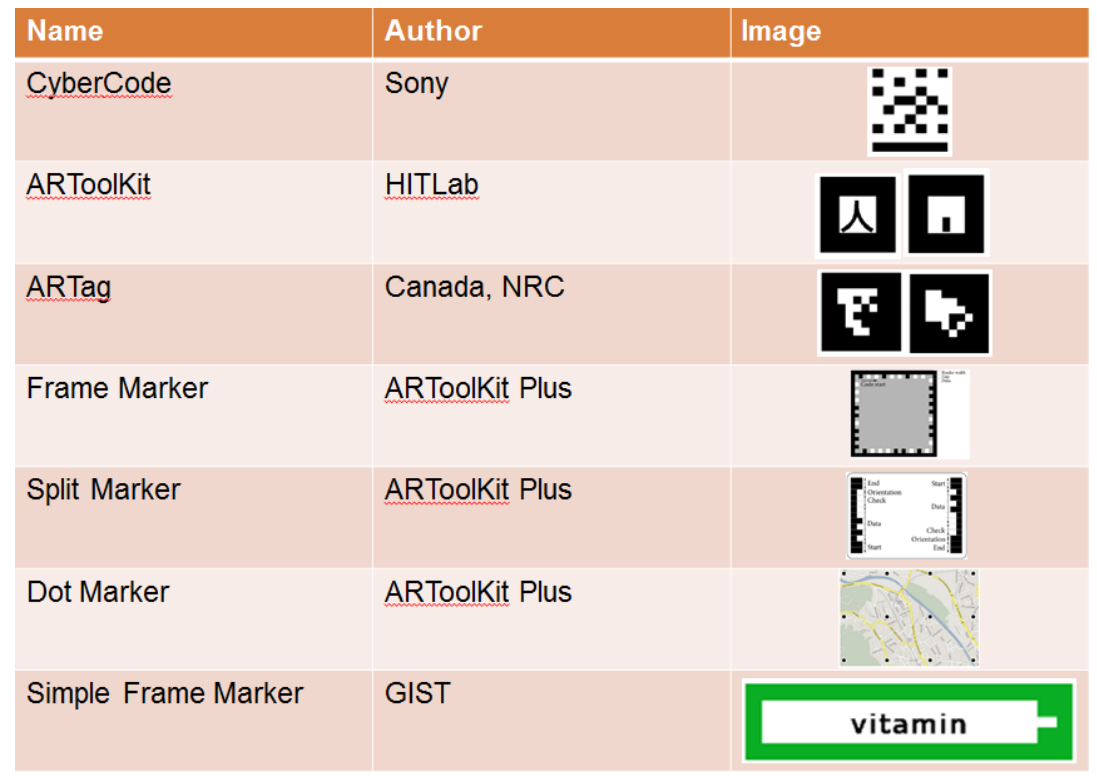

Fig. 1. Various AR Marker Systems

However, the problems of augment reality based on marker still existed even through these systems. In the Augment Reality based on marker, it predicts the 3D 
pose by using the specific limited numbers of points so the change of pose with video noises and there were jittering problems existed because of that. To solve this problem, there were some cases using the signal processing theory.[8] Nevertheless, these methods have a problem with the big amount of calculation and the unnaturalness of movement since it uses the history information to reduce the effect of noises. Additionally, without any tracking process, the Instability problem[9] which the object disappears even though marker exists when the marker fails the detection since it calculates the code area about the area where it is detected. This problem is considered as a serious problem on Interaction when the marker is covered with a hand.

In marker-less tracking, there are not exist these problems. Since markerless tracking technology uses many numbers of specific points for pose prediction and matching, the noise effect decreases through the average effect.[10] In addition, to improve the slow velocity during the process of matching, the tracking process applies and tracking can be provided strongly in the Detection Fail situation such as occlusion through many specific points and tracking technology.

\section{Proposed Method}

We propose a robust pose estimation method which uses multiple keypoints and an occlusion-reduction method which uses feature-tracking.

\subsection{Jittering Reduction Method}

The augment reality analyses the media, calculates the pose $\&$ the location of camera on World Coordinate System, and moves the object which will be augmented by moving the Graphics Camera based on this. For this, the augment reality extracts the feature points from video and estimates the camera's location and pose based on these feature points. As mentioned above, in previous marker tracking technology, it predicts the pose and location of camera by using only four vertexes of marker's most outer side. Because of video noise, the result of prediction can be changed and the jittering problem of augmented object occurred through this. The suggesting method reduces jittering through average effect by using the more feature points and hybrids the markerless tracking technology. Especially, many of the good points to select as the feature exists since each cell that are designed as digital cell (i.e. ARTag, ARToolKit Plus, etc) have a clear boundary. The suggesting method predicts the pose by using the boundary of cell as the feature points.

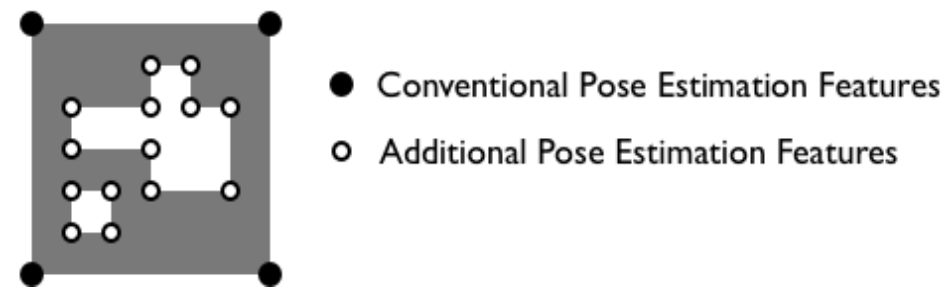

Fig. 2. Feature points which used to estimate the camera pose. Black discs represent conventional keypoints, and which dots are additional keypoints we adopted 
However, to predict the pose with additional points, the marker-coordinate of additional feature points have to be calculated. For this, the following steps are processed.

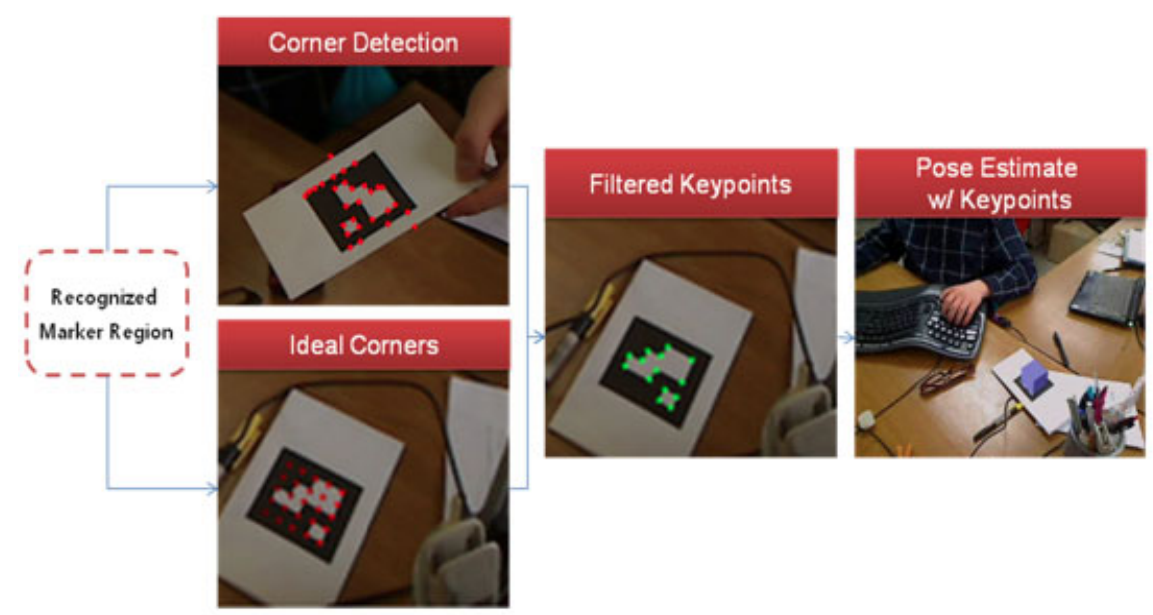

Fig. 3. Multiple keypoints based robust pose estimation process

It performs the corner detection on the area where it recognized as the marker. In this paper, we used [11] method. With this method, we can find the points that are strong in Cornerness on the image.

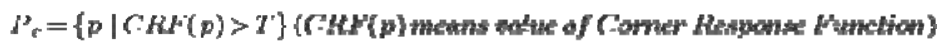

Additionally, it calculates the image coordinates of Ideal Corners in area that recognized as the marker. Ideal Corners are points that are located in the boundaries of cell in marker. It can be appeared on corner or edge or inside area depends on the ID. The marker- coordinates of Ideal corners are already known. The Ideal corners are transformed depends on the area of marker we can obtain the ideal corners that are transformed by the pose.

$$
1^{2}{ }_{i}=\{p \quad p \text { is an Ideal Corner, and } p \text { is transformed by warker region. }\}
$$

With matching the corners $\left(\mathrm{P}_{\mathrm{c}}\right)$ and ideal corners $\left(\mathrm{P}_{\mathrm{i}}\right)$ that are calculated with this method, we calculate the filtered keypoints $\left(\mathrm{P}_{\mathrm{f}}\right)$. These filtered keypoints are strong with cornerness on the image and able to know the marker coordinates that can be mapped.

$$
P_{f}=P_{r} \cap P_{i}
$$


Since these calculated feature points can know the image coordinate and marker coordinate, it can be used with pose prediction. With using the multiple points, it can predict the pose and can extract the pose prediction feature points

\subsection{Robust Marker Tracking Method}

The previous augmented reality based on markers augments an object by finding the marker with performing standard detection methods. These methods have disadvantages of disappearance of object because of detection failure, and the failure is occurred by several reason - e.g. occlusion, shadow, light change, etc. We solved this problem by adopting the feature tracking technology. The augmented reality toolkit operates in the input image, detects the marker, and keeps the detected feature points as the keypoints. When the detection failure occurs while the tracking, the KLT feature tracking algorithm is performed based on those keypoints already kept before, and pose is estimated by those tracked keypoints. Because this process is performed after a marker is detected in previous frame, the system can obtain the ID of marker and needs only feature points of the marker, so the augmentation can be worked continuously. Figure 4 shows the previous method (left), and the case when we keep tracking (right) when failure occurs.

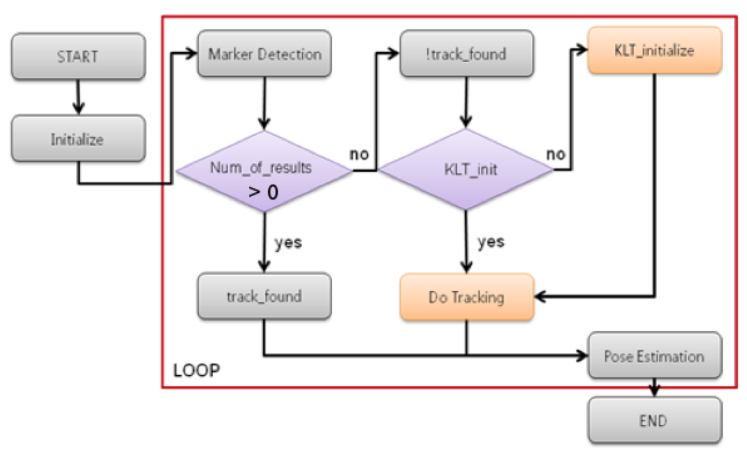

Fig. 4. Feature tracking process to overcome detection failure. Left comparison is same with conventional method, and the right comparison shows tracking method when failure occurs.

\section{Experiments}

To prove reduction jittering, we measured error of pose in 100 frames when the marker and the camera were still. ARToolKit adopts none jitter reduction method. Also ARToolKit Plus uses more robust pose estimation algorithm.

Table 1. Average error of pose in 100 still frames

\begin{tabular}{lll}
\hline ARToolKit & ARToolKit Plus & Proposed Method \\
\hline 3.24 & 2.76 & 0.98 \\
\hline
\end{tabular}


ARToolKit shows the most amount of error in 100 frames, and ARToolKit Plus provides less error. They, however, still larger than proposed method, because they took effect by image noise. In proposed method, we still suffer effect of noise, but the amount is less than others.

Also, to prove overcoming the occlusion problem, we performed the experiment from the paper of [9]. In [9], it called the case of failing the augmenting with occlusion in augment reality based on marker as corner case and defined as figure 5. In ARToolKit cases, it failed even though it is only occluded the one edge and ARTag failed when two side of edges were occluded.

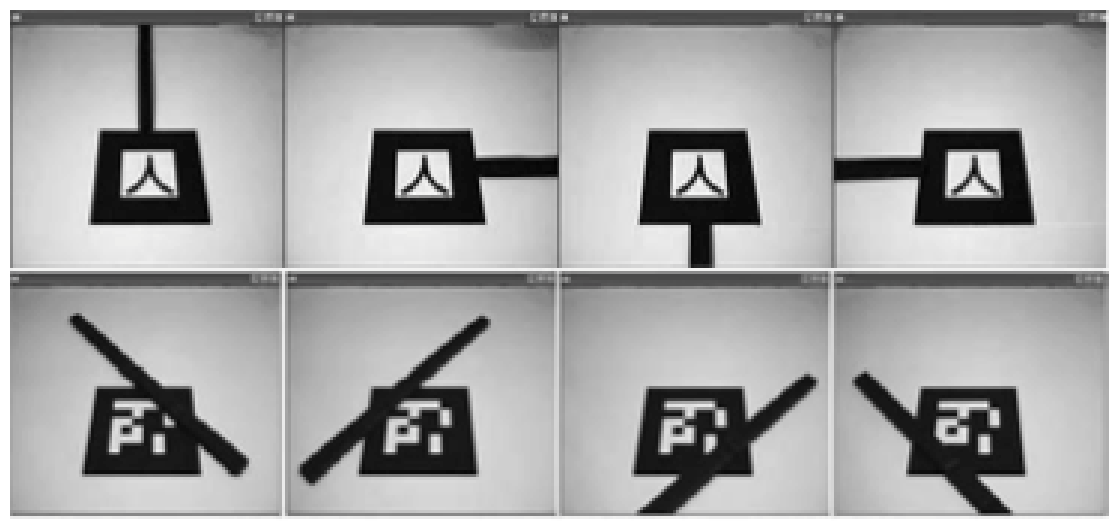

Fig. 5. Occlusion Corner Cases[9]. When edges are occluded, detection goes failure.

We applied the proposed method about those corner cases.

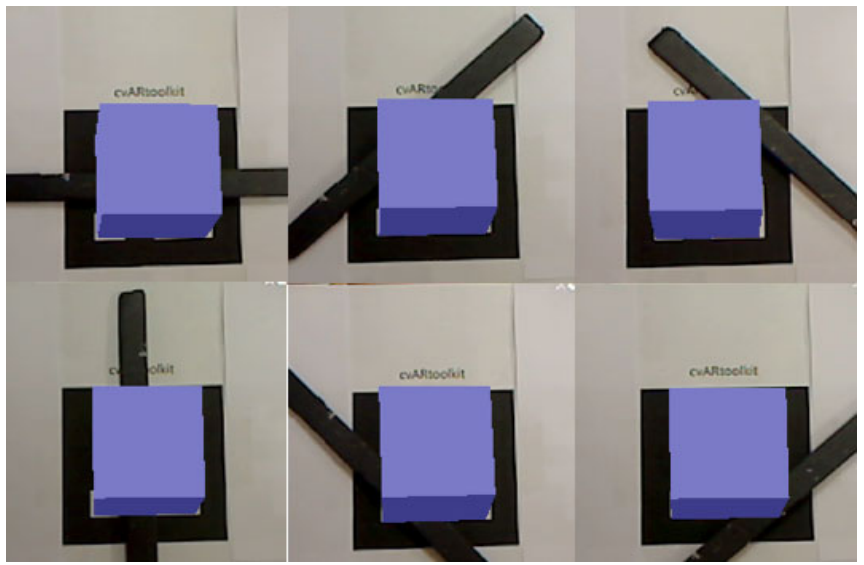

Fig. 6. Result of proposed method. It provides robust to occlusion. 
When we applied suggesting method, the marker was strongly does the tracking even though both edges were covered. However, since it does the tracking the feature points, there were some problems with the distorted shaped object when the feature points moves. This has to be solved as the future work.

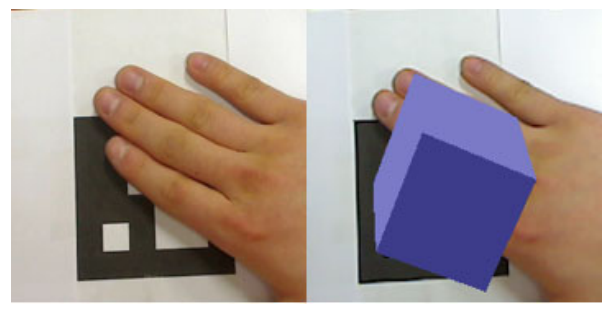

Fig. 7. Future work. When the tracking point is moves, augmented object is distorted.

\section{Conclusion}

In this paper, we have proposed two methods to overcome marker-based AR inherent problems - i.e. jittering and occlusion instability. When marker based AR is developed to commercial application, these problems are serious. We have solved these problem by hybrid with marker-less tracking technology. We implemented a multiple point based pose estimation method to reduce jitter, and adopted feature tracking method to overcome detection failure. In ordinary condition, they worked well, but, in some special condition, they need to be improved more.

Acknowledgement. This work (2010-0027654) was supported by Mid-career Researcher Program through NRF grant funded by the MEST.

\section{References}

1. Azuma, R.T.: A survey of augmented reality. Presence: Teleoperators and Virtual Environment 6(4), 355-385 (1997)

2. Milgram, P., Takemura, H., Utsumi, A., Kishino, F.: Augmented Reality: A class of displays on the reality-virtuality continuum. In: Proceedings of Telemanipulator and Telepresence Technologies, pp. 34-2351 (2007) (retrieved)

3. Rekimoto, J.: Matrix: A Realitime Object Identification and Registration Method for Augmented Reality. In: Proceeding of APCHI 1998, pp. 63-68 (1998)

4. Kato, H., Billinghurst, M.: Marker Tracking and HMD Calibration for a Video based Augmented Reality Conferencing System. In: Proceeding of IWAR 1999, pp. 85-94 (1999)

5. Fiala, M.: ARTag, a fiducial marker system using digital techniques. In: Proceedings of Computer Vision and Pattern Recognition, vol. 2, pp. 590-596 (2005)

6. Wagner, D., Langlotz, T., Schmalstieg, D.: Robust and unobtrusive marker tracking on mobile phones. In: Proceedings of ISMAR 2008, pp. 121-124 (2008)

7. Kim, H., Woo, W.: Simple Frame Marker for Image and Character Recognition. In: Proceedings of ISUV 2008, pp. 43-46 (2008) 
8. Rubio, M., Quintana, A., Pérez-Rosés, H., Quirós, R., Camahort, E.: Jittering Reduction in Marker-Based Augmented Reality Systems. In: Gavrilova, M.L., Gervasi, O., Kumar, V., Tan, C.J.K., Taniar, D., Laganá, A., Mun, Y., Choo, H. (eds.) ICCSA 2006. LNCS, vol. 3980, pp. 510-517. Springer, Heidelberg (2006)

9. Lee, S.-W., Kim, D.-C., Kim, D.-Y., Han, T.-D.: Tag Detection Algorithm for Improving a Instability Problem of an Augmented Reality. In: Proceedings of ISMAR 2006, pp. 257258 (2006)

10. Wagner, D., Schmalstieg, D., Bischof, H.: Multiple Target Detection and Tracking with Guaranteed Framerates on Mobile Phones. In: Proceedings of ISMAR 2009, pp. 57-64 (2009)

11. Harris, C., Stephens, M.: A Combined Corner and Edge Detector. In: Fourth Alvey Vision Conference, Manchester, UK, pp. 147-151 (1998) 